hep-th $/ 9707158$

IAS-97-84, RU-97-62

\title{
The Coulomb Branch of $(4,4)$ Supersymmetric Field Theories in Two Dimensions
}

\author{
Duiliu-Emanuel Diaconescu \\ Department of Physics and Astronomy \\ Rutgers University \\ Piscataway, NJ 08855-0849, USA \\ and \\ Nathan Seiberg \\ Institute for Advanced Study \\ Princeton, NJ 08540, USA
}

We study $(4,4)$ supersymmetric field theories in two dimensions with a one dimensional Coulomb branch. These theories have applications in string theory. Our analysis explains the known relation between $A-D-E$ groups and modular invariants of affine $S U(2)$. 


\section{Introduction}

Supersymmetric theories in various dimensions can be analyzed exactly. This has led to new insights in field theory and string theory. In particular, some theories with eight supercharges can be studied as toroidal compactifications of the minimal supersymmetric theories in six dimensions. This was done explicitly for compactifications to 5, 4 and 3 dimensions (see [1] and references therein). The purpose of this paper is to extend this discussion to compactification to 2 dimensions.

These field theories occur in string theory in different setups. First, they occur in the matrix model of M theory [2]. Second, they occur in compactifications on singular manifolds. Finally, they occur on various branes. The six dimensional theories are the low energy theories on the 5-branes of the heterotic theory. They can be viewed as systems of 5-branes and parallel 9-branes in M theory [3] or in IIB theory [4]. Then one can think of the background 9-branes as breaking 16 of the supersymmetries and the 5-branes can be viewed as probes [5]. Similarly, the five dimensional theories occur on 4-branes probing a background with 8-branes [6], the four dimensional theories occur on 3-branes probing a background with 7-branes [7], and the three dimensional theories occur on 2-branes probing a background with 6-branes [8]. Here we extend it to a 1-brane probing a background with 5-branes. Some aspects of this system have been discussed before [9] Finally, the system of 0-branes probing a background of 4-branes was studied in [10].

We limit ourselves to theories with a one dimensional Coulomb branch. These theories are classified by an $A-D-E$ type classification. The $A_{N_{f}-1}$ theories correspond to $U(1)$ gauge theories with $N_{f}$ electrons (their global symmetry includes an $S U\left(N_{f}\right)$ factor, hence the term $A_{N_{f}-1}$ theories). They correspond to a single $d$ dimensional probe in the presence of $N_{f} d+4$ dimensional background branes. The $D_{N_{f}}$ theories correspond to $S U(2)$ gauge theories with $N_{f}$ quarks (their global symmetry includes an $S O\left(2 N_{f}\right)$ factor, hence the term $D_{N_{f}}$ theories). They correspond to a single $d$ dimensional probe in the presence of $N_{f} d+4$ dimensional background branes and a $d+4$ dimensional orientifold. The other theories, including the various $E$ theories correspond to background branes at strong coupling.

There is a crucial difference between the higher dimensional field theories and the two or one dimensional cases. Here, the notion of moduli space of vacua is ill defined. One should integrate over all the vacua. However, one intuitively expects, in the spirit of the Born-Oppenheimer approximation, a notion of a low energy theory obtained by integrating 
out the high energy modes, which looks like a non-linear sigma model on a target space. We will refer to this target space as the moduli space of vacua.

As in the higher dimensional analogs, these systems have a Coulomb branch of vacua corresponding to motion of the probe away from the background branes. The Higgs branch corresponds to the absorption of the probe in the background branes as an instanton [11]. The theory on this branch was used recently in [12,13] to give a description of the $(2,0)$ field theory [14,15] and string theory [16]. As in [17], we can promote the gauge coupling to a background superfield. In our case the gauge coupling is in a vector superfield [18], and therefore the Higgs branch is not corrected by quantum effects. The Coulomb branch can be corrected.

Here we will focus on theories with a one dimensional Coulomb branch. Its metric turns out to be completely determined by the global symmetries of the problem and a simple one loop computation. This metric leads to two separate problems. First, in most cases the Coulomb branch develops an infinitely long tube. It is not completely clear how to interpret the physics along the tube. More importantly, in some cases (e.g. in an $S U(2)$ gauge theory without quarks) the metric has a singularity at a finite distance on the moduli space beyond which the metric ceases to be Riemannian. This situation is reminiscent of similar problems in four [19,20] and three [8,21] dimensions. In both cases the problem is solved by realizing that the degrees of freedom used to describe the Coulomb branch are not appropriate and one needs to dualize them. In terms of the dual degrees of freedom there exists a Riemannian metric everywhere on the moduli space.

We find the generation of the infinitely long tube counter intuitive. It means that the wave function of the light modes is not normalizable near the origin (it is also not normalizable far out along the flat direction). This is confusing because classically this region is compact. Therefore, we speculate that there exists another description of the field theory near the origin, which is compact there in the sense that the wave function is normalizable. Some evidence for this speculation comes from the second problem mentioned above which suggests that in some, yet to be found, "dual variables" there is no problem with a non-Riemannian metric. In the following sections we will consider the compactifications of these theories from three to two dimensions and will find further evidence for this speculation.

In the context of string theory we find these theories on D1-branes near D5-branes and orientifolds. Therefore, we find them in IIB compactifications on $T^{4} / Z_{2}$. A careful examination of the action of the $Z_{2}$ shows that this theory is dual to a compactification 
of IIA on K3 [22]. The Coulomb branch of the $1+1$ dimensional theory is then mapped, after T-duality, to the underlying K3. This space does not have any long tubes. Instead, its singularities are classified by $A-D-E$. Therefore, it is reasonable to expect that in some dual variables the region around the origin can be replaced by an ALE space with an $A-D-E$ singularity. Such a proposal has already been made in [23] (see however, [24]). It should be stressed that the $1+1$ dimensional theory on the ALE space is not merely an orbifold theory because the $\theta$ angle there vanishes rather than being equal to $\pi$ [25].

In section 2 we present the general formalism for describing these systems. In section 3 we study $U(1)$ gauge theories with $N_{f}$ electrons and in section 4 we study $S U(2)$ gauge theories with $N_{f}$ quarks. In section 5 we discuss three theories, which do not have a simple gauge theory Lagrangian, whose global symmetries are $E_{6,7,8}$. In section 6 we focus on the region in the moduli space near the origin - the tube - and present an explanation of the known connection between $A-D-E$ symmetries and the classifications of modular invariants of $\widehat{S U(2)}[26]$.

\section{Preliminaries}

The $\mathcal{N}=(4,4)$ supersymmetry algebra in two dimensions is the dimensional reduction of the six dimensional $\mathcal{N}=(1,0)$. The supersymmetry charges of the latter are symplectically real chiral spinors transforming as the $\mathbf{4}$ of $\operatorname{Spin}(1,5)$ and as the doublet of the R-symmetry group $S U(2)_{R}$. Dimensional reduction to two dimensions yields eight real supercharges in

$$
\begin{aligned}
(\mathbf{2}, \mathbf{1}, \mathbf{2})^{+} \oplus(\mathbf{1}, \mathbf{2}, \mathbf{2})^{-} \quad \text { of } \quad & \operatorname{Spin}(4) \times S U(2)_{R} \times S O(1,1) \\
& \subset \operatorname{Spin}(1,5) \times S U(2)_{R}
\end{aligned}
$$

(where the representations of $\operatorname{Spin}(4)$ are labeled by their two $S U(2)$ subgroups). As in six dimensions, this algebra has two massless representations: a vector multiplet and a hypermultiplet. In two dimensions these two representations are related by duality (more about this below).

A hypermultiplet contains four scalars transforming as $(\mathbf{1}, \mathbf{1}, \mathbf{2}) \oplus(\mathbf{1}, \mathbf{1}, \mathbf{2})$ under $\operatorname{Spin}(4) \times S U(2)_{R}$ and four fermions. In terms of an $\mathcal{N}=(2,2)$ subalgebra the hypermultiplet decomposes into two chiral multiplets $\Phi$ and $\widetilde{\Phi}$ satisfying

$$
\begin{array}{ll}
\bar{D}_{+} \Phi=0, & \bar{D}_{-} \Phi=0 \\
\bar{D}_{+} \widetilde{\Phi}=0, & \bar{D}_{-} \widetilde{\Phi}=0 .
\end{array}
$$


The scalars in these multiplets parameterize a "Higgs branch," which is a hyper-Kähler manifold. The three complex structures are in the $\mathbf{3}$ of $S U(2)_{R}$. Along the Higgs branch there are scalars transforming in $\mathbf{3} \oplus \mathbf{1}$ of $S U(2)_{R}$. The $\operatorname{Spin}(4)$ global symmetry does not act on the Higgs branch but it acts on the fermions.

The vector multiplet is also known as a twisted multiplet. In terms of an $\mathcal{N}=$ $(2,2)$ subalgebra it decomposes into a chiral multiplet $\Phi$ and a twisted chiral multiplet $\Lambda$ satisfying

$$
\begin{array}{ll}
\bar{D}_{+} \Phi=0, & \bar{D}_{-} \Phi=0 \\
D_{+} \Lambda=0, & \bar{D}_{-} \Lambda=0 .
\end{array}
$$

The four real scalars contained in these superfields represent the components of the six dimensional vector field $A_{\mu}$ along the reduced directions. They transform as $(\mathbf{2}, \mathbf{2}, \mathbf{1})$ of $\operatorname{Spin}(4) \times S U(2)_{R}$. These scalars parameterize the "Coulomb branch." It is characterized [27] by a generalized Kähler potential $K(\Lambda, \Phi)$ satisfying the Laplace equation

$$
K_{\Lambda \bar{\Lambda}}+K_{\Phi \bar{\Phi}}=0
$$

It determines the metric

$$
d s^{2}=K_{\Phi \bar{\Phi}} d \Phi d \bar{\Phi}-K_{\Lambda \bar{\Lambda}} d \Lambda d \bar{\Lambda}
$$

and the torsion

$$
B=\frac{1}{4}\left(K_{\Phi \bar{\Lambda}} d \Phi \wedge d \bar{\Lambda}+K_{\Lambda \bar{\Phi}} d \bar{\Phi} \wedge d \Lambda\right)
$$

Note that the metric is not hyper-Kähler. The $\mathcal{N}=(4,4)$ supersymmetry is realized in terms of three complex structures, which are covariantly constant with respect to the generalized connection with torsion. Unlike the Higgs branch, here $S U(2)_{R}$ does not act on the space. Instead, the Spin(4) group acts on it and is spontaneously broken to a subgroup at a generic point.

If the bosonic manifold defined by $(2.5)$ admits a compact $U(1)$ isometry generated by the Killing vector $i(\partial / \partial \Lambda-\partial / \partial \bar{\Lambda})$, we can dualize the twisted superfield $\Lambda$ to an ordinary chiral superfield $\chi$. This breaks the $\operatorname{Spin}(4) \times S U(2)_{R}$ invariance of the action to $S U(2)_{L} \times S U(2)_{R}$ where $S U(2)_{L} \subset \operatorname{Spin}(4)$ and there is an extra $U(1)$ symmetry corresponding to the isometry. The $\mathcal{N}=(4,4)$ sigma model with torsion is dual to a hyper-Kähler sigma model with isometry group $S U(2)_{L} \times U(1)$ identical to the one spanned by the hypermultiplets. Note that manifest $\mathcal{N}=(4,4)$ invariance is preserved by this transformation, if and only if the compact $U(1)$ isometry is translational, i.e. it preserves the three complex structures. 
The action may also contain bare parameters: mass terms for the matter hypermultiplets transforming as $(\mathbf{2}, \mathbf{2}, \mathbf{1})$ of $\operatorname{Spin}(4) \times S U(2)_{R}$ and Fayet-Iliopoulos terms in the $(\mathbf{1}, \mathbf{1}, \mathbf{3})$ representation. Taking into account the $\mathcal{N}=(4,4)$ supersymmetry constraints and the transformation properties under global symmetries we can prove certain nonrenormalization theorems analogous to those in [18]:

1. The gauge coupling constant can be promoted to a background vector superfield, thus it can affect only the metric on the Coulomb branch and not the Higgs branch.

2. The mass terms can also be regarded as scalar components of vector superfields, thus they can affect only the Coulomb branch but not the metric on the Higgs branch.

3. The FI parameters can be promoted to hypermultiplets, thus they can only appear in the metric on the Higgs branch.

At long distance the theory must be a superconformal field theory 1 . The standard $\mathcal{N}=$ 4 superconformal algebra includes an $S U(2)$ factor. Therefore, we expect at long distance an $S U(2) \times S U(2)$ symmetry under which the left moving supercharges are in $(\mathbf{2}, \mathbf{1})$ and the right moving supercharges in $(\mathbf{1}, \mathbf{2})$. As the theory on the Higgs branch is a sigma model on a hyper-Kähler manifold, the $S U(2) \times S U(2)$ symmetry in the superconformal algebra should not act on the bosons. Therefore, we immediately identify this $S U(2) \times S U(2)$ symmetry as the Spin(4). The situation on the Coulomb branch is more complicated for two related reasons. First, the Spin(4) symmetry acts on the bosons, and second it is not a hyper-Kähler manifold. In section 6 we will discuss the superconformal algebra on the Coulomb branch and show that it includes the $S U(2)_{R}$ symmetry. Considerations similar to these have led Witten [14,13] to suggest that the Coulomb branch is decoupled from the Higgs branch.

\section{3. $A_{N_{f}-1}$ Theories $-U(1)$ with $N_{f}$ electrons}

In this section we consider $U(1)$ gauge theories with $N_{f}$ electrons. The global symmetry of these theories is $S U\left(N_{f}\right) \times \operatorname{Spin}(4) \times S U(2)_{R}$.

For $N_{f}=0$ the theory is free. For $N_{f} \geq 1$ the gauge coupling, is a relevant operator. The theory has a Coulomb branch parameterized by the expectation values $\vec{r} \in R^{4}$ of the

1 Since the target space of the low energy effective theory on the moduli space is noncompact, it is possible that the theory is scale invariant but not conformally invariant. 
scalars in the twisted multiplet. The tree level metric is flat and the torsion vanishes. Equivalently,

$$
K_{c l}(\Phi, \bar{\Phi}, \Lambda, \bar{\Lambda})=\frac{1}{g_{2}^{2}}(\Phi \bar{\Phi}-\Lambda \bar{\Lambda}) .
$$

Quantum mechanically $K$ can be corrected. In general, the geometry of the target manifold is entirely determined by the function

$$
f(\Phi, \bar{\Phi}, \Lambda, \bar{\Lambda})=K_{\Phi \bar{\Phi}}=-K_{\Lambda \bar{\Lambda}}
$$

which clearly satisfies the Laplace equation:

$$
f_{\Phi \bar{\Phi}}+f_{\Lambda \bar{\Lambda}}=0
$$

This equation has a unique $\operatorname{Spin}(4)$ invariant solution given by

$$
f=A+\frac{k}{\Phi \bar{\Phi}+\Lambda \bar{\Lambda}}
$$

for some constants $A$ and $k$. (This fact is a special case of a more general result in $\mathcal{N}=(0,4)$ supersymmetric theories [28].) We find it convenient to assign dimension one to all the scalars (as they are components of gauge fields in six dimensions). Then the gauge coupling $g_{2}$ has dimension one. Therefore, $A$ in (3.4) can only appear at tree level and $A=\frac{1}{g_{2}^{2}}$. Similarly, $k$ can only appear at one loop. No higher order perturbative or non-perturbative corrections of $K$ are possible.

An explicit one loop computation determines the value of $k=N_{f}$. Therefore, the exact generalized Kähler potential is [29]

$$
K(\Phi, \bar{\Phi}, \Lambda, \bar{\Lambda})=\frac{1}{g_{2}^{2}}(\Phi \bar{\Phi}-\Lambda \bar{\Lambda})-N_{f} \int^{\frac{\Lambda \bar{\Lambda}}{\Phi \bar{\Phi}}} \frac{d \xi}{\xi} \ln (\xi+1)+\ln \Phi \ln \bar{\Phi}
$$

This leads to the metric

$$
d s^{2}=\left(\frac{1}{g_{2}^{2}}+\frac{N_{f}}{r^{2}}\right) d \vec{r}^{2}
$$

and torsion

$$
B=-\frac{1}{4} N_{f} \sin ^{2} \frac{\theta}{2} d \phi \wedge d \chi
$$

where $0 \leq \theta<\pi, 0 \leq \phi<2 \pi, 0 \leq \chi<4 \pi$ are angular coordinates on the unit three sphere $S^{3} \subset R^{4}$

$$
\Phi=e^{i(\chi-\phi) / 2} \cos \frac{\theta}{2}, \quad \Lambda=e^{i(\chi+\phi) / 2} \sin \frac{\theta}{2}
$$


Strictly speaking, the two-form is not globally defined on $S^{3}$, similarly to the Dirac monopole vector potential, but it yields a well defined three-form field strength proportional to the volume form of the sphere:

$$
H=-N_{f} d \Omega^{3}
$$

It is possible to turn on bare masses $\vec{m}_{i}, i=1, \ldots, N_{f}$ for the hypermultiplets (one of the masses can be set to zero by a choice of the origin on the Coulomb branch). The global Spin(4) symmetry is explicitly broken and the one-loop corrections on the Coulomb branch are

$$
\begin{aligned}
d s^{2} & =\left(\frac{1}{g_{2}^{2}}+\frac{1}{\left|\vec{r}-\vec{m}_{1}\right|^{2}}+\frac{1}{\left|\vec{r}-\vec{m}_{2}\right|^{2}}+\ldots+\frac{1}{\left|\vec{r}-\vec{m}_{N_{f}}\right|^{2}}\right) d \vec{r}^{2} \\
H & =-d \Omega_{1}^{3}-\ldots-d \Omega_{N_{f}}^{3}
\end{aligned}
$$

where $\vec{r}=(\Phi, \Lambda)$ and $d \Omega_{i}^{3}$ is the volume form on the unit sphere centered around the point of coordinates $\vec{m}_{i}$. The behavior of $f$ near a particular point $\vec{m}_{i}$ is determined to be of the form (3.4) by decoupling the remaining hypermultiplets $\left(\vec{m}_{j} \rightarrow \infty, j \neq i\right)$. This result is exact, since the function $f(\Phi, \bar{\Phi}, \Lambda, \bar{\Lambda})$ satisfies the Laplace equation in $R^{4} \backslash\left\{\vec{m}_{1}, \ldots, \vec{m}_{N_{f}}\right\}$ with fixed boundary conditions at infinity and around the singular points $\vec{m}_{i}$.

In section 6 we will analyze the singularities in the metric in more detail. Here we simply note that the metric near the singularities has an infinitely long tube.

The Higgs branch of these models depends on the number of massless flavors. As explained in section 2, the metric on this space is completely fixed at the classical level by a hyper-Kähler quotient construction and is not corrected quantum mechanically. For $N_{f}=1$ there is no Higgs branch. For higher values of $N_{f}$ the Higgs branch is isomorphic to the moduli space of a single $S U\left(N_{f}\right)$ instanton in $R^{4}$. The singularity at the origin corresponds to a point-like instanton.

We now consider the corresponding $\mathcal{N}=4$ theories on $R^{2} \times S_{R}^{1}$, where the circle is of radius $R$. This should interpolate between the three dimensional results of [8,21] and the two dimensional theory. In three dimensions electric-magnetic duality transforms the vector multiplet into a hypermultiplet. The metric is then constrained by symmetries and asymptotic one loop corrections to be the Taub-NUT metric. Consider first the case where the radius $R$ is much larger than the distance scale set by the three dimensional gauge coupling $g_{3}$. Then, at energies above $1 / R$ the theory is three dimensional and the renormalization group evolution leads to the Taub-NUT metric. At energies below $1 / R$ 
the renormalization group evolution is that of the two dimensional sigma model based on this space. Since this sigma model is conformally invariant, it does not evolve further and the Coulomb branch ends up being the Taub-NUT space with an $A_{N_{f}-1}$ singularity (we expect the $\theta$ angle at the singularity to vanish such that the $1+1$ dimensional theory is not an orbifold theory [25]). Note that one of the degrees of freedom on this space is dual to the natural variables in the two dimensional theory - the space is parametrized by a hypermultiplet rather than by a vector multiplet. This is an explicit realization of the speculation in the introduction.

We now compute the exact one loop corrected metric on $R^{2} \times S_{R}^{1}$ for any $R$. The classical moduli space is $R^{3} \times S^{1}$ where the $S^{1}$ factor is the Wilson line around the compact dimension. It is parameterized by the component of the three dimensional gauge field $\sigma \sim \sigma+\frac{1}{R}$. The three scalars in $R^{3}$ are denoted by $\vec{\phi}$. Standard dimensional reduction relates the three and the two dimensional coupling constants

$$
\frac{1}{g_{2}^{2}}=\frac{2 \pi R}{g_{3}^{2}}
$$

The relevant diagram is the vacuum polarization diagram with hypermultiplets running in the loop

$$
\sum_{n=-\infty}^{+\infty} \int \frac{d^{2} p}{(2 \pi)^{2} R} \frac{1}{\left(p^{2}+\vec{\phi}^{2}+\left(\frac{n}{R}+\sigma\right)^{2}\right)^{2}}
$$

Using standard Poisson resummation techniques we find the one loop corrected gauge coupling包

$$
\frac{1}{g_{2}^{2}}+N_{f} \frac{2 \pi R}{|\vec{\phi}|}\left\{\frac{1}{2}+\sum_{n \geq 1} e^{-2 \pi R n|\vec{\phi}|} \cos (2 \pi R n \sigma)\right\}
$$

This result interpolates smoothly between the three dimensional and the two dimensional regime. When the compactification radius is large $R \gg \frac{1}{|\vec{\phi}|}$ the exponential corrections are suppressed and the result reduces to the three dimensional one

$$
\frac{1}{g_{2}^{2}}+\pi R \frac{N_{f}}{|\vec{\phi}|}
$$

2 In the original version of this paper the following equation had an error. We thank J. Harvey and G. Moore for pointing this out to us. 
For small radius $R \ll \frac{1}{|\vec{\phi}|}$ the sum can be approximated by an integral leading to the two dimensional result (3.6)

$$
\frac{1}{g_{2}^{2}}+\frac{N_{f}}{|\vec{\phi}|^{2}+\sigma^{2}}
$$

The answer (3.13) demonstrates the difficulty in proving the speculation in the introduction. In the three dimensional theory $\sigma$ is a component of a vector field. The Taub-NUT metric is obtained after it is dualized to a scalar $\widetilde{\sigma}$. The three dimensional theory has a global (magnetic) $U(1)$ symmetry under which $\widetilde{\sigma}$ is shifted by a constant. If we dimensionally reduce the tree level theory, then $\sigma$ and $\widetilde{\sigma}$ are related by ordinary T-duality. However, the one loop corrected metric (3.13) shows that the compactified theory no longer has a continuous symmetry shifting $\sigma$ by a constant. Therefore, it is not clear how to dualize it. Similar problems of T-duality with respect to transformations, which are not symmetries, are familiar in two dimensions [30].

In the three dimensional theory every mass term has three real components in $(\mathbf{3}, \mathbf{1})^{0}$ of the global $S U(2)_{L} \times S U(2)_{R} \times U(1)$. After the compactification to two dimensions there are four real parameters. The fourth one arises as a Wilson line of the corresponding global symmetry around the circle. More generally, starting in six dimensions and compactifying on a four torus, all four mass parameters arise as Wilson lines. With non-zero masses in three dimensions and Wilson lines the one loop result becomes

$$
\frac{1}{g_{2}^{2}}+\sum_{i=1}^{N_{f}} \frac{2 \pi R}{\left|\vec{\phi}-\vec{m}_{i}\right|}\left\{\frac{1}{2}+\sum_{n \geq 1} e^{-2 \pi R n\left|\vec{\phi}-\vec{m}_{i}\right|} \cos \left[2 \pi R n\left(\sigma-\sigma_{i}\right)\right]\right\}
$$

This two dimensional field theory appears as the effective theory on a D1-brane near $N_{f}$ D5-branes. The $U(1)$ vector multiplet arises from strings, whose two ends touch the D1-brane, and the $N_{f}$ electrons arise from strings, which connect the D1-brane to the D5branes. The Coulomb branch corresponds to the situation, where the D1-brane is separated from the D5-branes but is parallel to them. The metric (3.6) is the standard metric near a D5-brane obtained by solving the classical supergravity equations [9]. Similarly, the torsion (3.7) represents the $B$ field generated by the charge of the D5-branes.

We can also study a single D2-brane and $N_{f}$ D6-branes wrapping a circle of radius $R$. The effective theory corresponding to this system is the three dimensional gauge theory compactified on a circle which we discussed above. 


\section{4. $D_{N_{f}}$ Theories $-S U(2)$ with $N_{f}$ flavors}

Here we study $S U(2)$ gauge theories with $N_{f}$ quark flavors in the fundamental representation. The global symmetry of these theories is $S O\left(2 N_{f}\right) \times \operatorname{Spin}(4) \times S U(2)_{R}$. The analysis of these theories is similar to the discussion in the previous section with some minor differences.

Unlike the $U(1)$ theories the Coulomb branch is not $R^{4}$ but $R^{4} / Z_{2}$ where the $Z_{2}$ originates from the Weyl group of $S U(2)$. The classical metric on this space is flat. As in the $U(1)$ theories, the global $\operatorname{Spin}(4)$ symmetry completely determines the quantum metric up to one coefficient which is easily computed at one loop

$$
d s^{2}=\left(\frac{1}{g_{2}^{2}}+\frac{2 N_{f}-2}{r^{2}}\right) d \vec{r}^{2}
$$

(the -2 represents the contribution of the non-Abelian vector multiplet). The corresponding torsion is

$$
H=-2\left(N_{f}-1\right) d \Omega^{3} .
$$

The cases $N_{f}=0,1$ are particularly interesting. For $N_{f}=0$ the metric has a singularity at finite distance in the moduli space $r_{0}=g_{2} \sqrt{2}$ and becomes negative definite past this value of $r$. This signals the fact that the present variables are not adequate to describe the physics in that region. For $N_{f}=1$ the effects of the charged hypermultiplet cancel precisely those of the non-Abelian vector multiplet and the metric remains flat and there is no tube in the metric. Similar phenomena occur in $S U(2)$ gauge theory with $N_{f}=2^{d-2}$ in $d=3,4,5,6$ dimensions.

Adding masses for hypermultiplets explicitly breaks the Spin(4) symmetry and results in quantum corrections of the form

$$
\begin{aligned}
d s^{2} & =\left(\frac{1}{g_{2}^{2}}+\frac{1}{\left|\vec{r}-\vec{m}_{1}\right|^{2}}+\frac{1}{\left|\vec{r}+\vec{m}_{1}\right|^{2}}+\ldots+\frac{1}{\left|\vec{r}-\vec{m}_{N_{f}}\right|^{2}}+\frac{1}{\left|\vec{r}+\vec{m}_{N_{f}}\right|^{2}}-\frac{2}{r^{2}}\right) d \vec{r}^{2} \\
H & =-d \Omega_{1-}^{3}-d \Omega_{1+}^{3}-\ldots-d \Omega_{N_{f}-}^{3}-d \Omega_{N_{f}+}^{3}+2 d \Omega^{3}
\end{aligned}
$$

where $d \Omega_{i \pm}^{3}$ is the volume form on the unit sphere centered around the point of coordinates $\pm \vec{m}_{i}$. As before, this metric is exact.

The Higgs branch is again determined at the classical level by hyper-Kähler quotient constructions and is not corrected quantum mechanically. It is isomorphic to the moduli space of a single $S O\left(2 N_{f}\right)$ instanton in $R^{4}$ (there is no Higgs branch for $N_{f}=0,1$ ). 
In three dimensions the Coulomb branch of these theories can be described in hypermultiplet variables. For $N_{f} \geq 2$ the metric is determined at one loop to be Taub-NUT [21]. For $N_{f}=0,1$ there are instanton corrections resulting in the Atiyah-Hitchin metric 21]. Reduction to two dimensions on a large circle yields an $\mathcal{N}=(4,4)$ sigma model with the corresponding hyper-Kähler metric. As in the $U(1)$ case, this metric leads to a scale invariant two dimensional field theory with $D_{N_{f}}$ singularity (we expect the $\theta$ angle at the singularity to vanish such that the $1+1$ dimensional theory is not an orbifold theory [25]). Again, we see an explicit realization of the speculation in the introduction. For the special cases $N_{f}=0,1$ the metric is not singular and the problem of the sign of the metric for $N_{f}=0$ is absent. Unfortunately, we do not know how to extend this discussion to smaller $R$ essentially for the reasons explained in the previous section (the compactified theory does not have the global symmetry needed for the T-duality).

The brane realization of these $S U(2)$ theories is achieved on a D1-brane near $N_{f}$ D5-branes and an orientifold. The asymptotic transverse space is $R^{4} / Z_{2}$ and the one loop corrected metric (4.1) is the spacetime metric obtained by solving the classical supergravity equations. Similarly, the torsion (4.2) represents the $B$ field generated by the charge of the orientifold and the $N_{f}$ D5-branes - charge -2 from the orientifold and $+2 N_{f}$ from the D5-branes. Again, the Coulomb branch corresponds to the D1-brane away from the D5branes and the orientifold and the Higgs branch represents the absorption of the D1-brane in them as an instanton.

\section{5. $E_{6,7,8}$}

In higher dimensions there exist other theories with a one dimensional Coulomb branch. Their global symmetries are $E_{6,7,8}$. They do not have $E_{6,7,8}$ invariant Lagrangians, which flow to them 3 . When we compactify them on tori to two dimensions we should find two dimensional theories with these global symmetries. We take such a compactification as the definition of the two dimensional theories.

The Higgs branches of these theories in higher dimensions are isomorphic to the moduli spaces of a single $E_{6,7,8}$ instanton in $R^{4}$. Since the parameters of the compactification are in vector multiplets, they cannot affect the Higgs branches. Therefore, the Higgs branches

3 In three dimensions they can be found in the long distance limit of local field theories based on Lagrangians, which are not $E_{6,7,8}$ invariant [31. 
of these theories in two dimensions are also isomorphic to the moduli spaces of an $E_{6,7,8}$ instanton in $R^{4}$.

Since we do not have a Lagrangian description of these theories, we cannot analyze the region far out along the Coulomb branch. In the next section we will describe these theories near the origin.

In string theory these theories occur on a D1-brane probing a background of 5-branes at strong coupling.

\section{The Theory in The Tube}

In this section we consider the region in the Coulomb branch around the origin. If we consider a fixed region around a point $\vec{r}$ in the moduli space and take the strong coupling limit $g_{2} \rightarrow \infty$ (recall that we define the fields such that there is a factor of $\frac{1}{g_{2}^{2}}$ in front of the action), the metric and torsion become

$$
\begin{aligned}
& d s^{2}=k \frac{d \vec{r}^{2}}{r^{2}} \\
& H=-k d \Omega^{3}
\end{aligned}
$$

where $k$ denotes the total $B$-field charge

$$
k=\left\{\begin{array}{cl}
N_{f} & \text { for } U(1) \\
2\left(N_{f}-1\right) & \text { for } S U(2)
\end{array}\right.
$$

For $S U(2)$ with $N_{f}=1, k=0$, and therefore the metric on the Coulomb branch is flat and proportional to $\frac{1}{g_{2}^{2}}$. For $S U(2)$ with $N_{f}=0$ the metric we find is not Riemannian, and therefore there must be a new description of the theory.

For $k \geq 1$, a change of variables $\phi=\sqrt{k} \ln \sqrt{r^{2} / k}$ yields the familiar tube [9]

$$
\begin{aligned}
& d s^{2}=d \phi^{2}+k d s_{3}^{2}, \\
& H=-k d \Omega^{3}
\end{aligned}
$$

where $d s_{3}^{2}, d \Omega^{3}$ are the metric and the volume form on the unit three sphere $S^{3} \subset R^{4}$. The metric and anti-symmetric tensor field on $S^{3}$ define a level $k$ supersymmetric $S U(2)$ WZW model and the non-trivial dilaton defines a supersymmetrized Liouville field with background charge

$$
Q=\sqrt{\frac{2}{k}} .
$$


For the $D_{N_{f}}$ theories the WZW model is defined on the group manifold $S O(3) \sim S^{3} / Z_{2}$ rather than on its double cover, reflecting the $Z_{2}$ action on the Coulomb branch. This is consistent with the level of the corresponding affine algebra being even.

The supersymmetric WZW theories are analyzed by first performing a chiral rotation making the fermions free. This has the effect of shifting the coefficient of the WZ term for the bosons $k \rightarrow \widetilde{k}=k-2$. One way to understand this is to note that the three free fermions lead to an $\widehat{S U(2)_{2}}$ algebra and the bosons to $\widehat{S U(2)} \widetilde{k}_{k=k-2}$, which is generated by $J_{i}$. The diagonal affine algebra has level $\widetilde{k}+2=k$. Therefore, for $k>2$ we are left with a WZW theory with level $\widetilde{k}>0$. For the two cases with $k=2$ (the $A_{1}$ and the $D_{2}$ theories) there is no bosonic WZW theory - the resulting theory has only the boson $\phi$ in (6.3) and four free fermions. For $k=1$ (the $A_{0}$ theory) $\widetilde{k}<0$. Therefore, the supersymmetric WZW theory is not unitary and a new description of the theory should be found.

The four free fermions $\psi^{a}$ ( $a=0$ for the superpartner of $\phi$ and $a=1,2,3$ for the free fermions obtained by the chiral rotation ) lead to an $\widehat{S U(2)} \times \widehat{S U(2)}$, algebra generated by

$$
j_{i}^{ \pm}=\epsilon_{i j k} \psi^{j} \psi^{k} \mp \psi^{0} \psi^{i}
$$

(the previously mentioned $\widehat{S U(2)_{2}}$ is the diagonal subalgebra). At this stage we can compare the various symmetries with the classical $\operatorname{Spin}(4) \times S U(2)_{R}$ symmetry of our theory. From the action of the $\operatorname{Spin}(4) \cong S U(2) \times S U(2)$ on the supercharges it is clear that one $S U(2)$ leads to a left moving symmetry and the other $S U(2)$ to a right moving symmetry. Since it acts on the bosons, the corresponding current must include $J_{i}$. The anomaly in this current can be computed semiclassically [13]. The fermions in the hypermultiplets contribute 1 and the fermions in the vector multiplets contribute -1 . Therefore, the anomaly is $N_{f}-1=k-1$ in the $A_{N_{f}-1}$ theories and $2 N_{f}-3=k-1$ in the $D_{N_{f}}$ theories. The $S U(2)_{R}$ symmetry does not act on the bosons on the Coulomb branch. Therefore, it must be extended in the long distance theory to two $S U(2)$ symmetries - one for the left movers and one for the right movers. The four right moving supercharges should therefore be in $(\mathbf{2}, \mathbf{2})$ of an $S U(2)$ from the $\operatorname{Spin}(4)$ and a new $S U(2)$ which does not act on the bosons. This together with the anomalies leads to the identification of these two $\widehat{S U(2)}$ as being generated by

$$
\begin{aligned}
& A_{i}^{+}=j_{i}^{+}+J_{i} \\
& A_{i}^{-}=j_{i}^{-} .
\end{aligned}
$$


Using these currents and the energy momentum and supercurrents

$$
\begin{aligned}
& T=-J^{0} J^{0}-\frac{1}{k} J^{i} J^{i}-\partial \psi^{a} \psi^{a} \\
& G^{0}=2\left\{J^{0} \psi^{0}+\frac{1}{\sqrt{k}} J^{i} \psi^{i}+\frac{2}{\sqrt{k}} \psi^{1} \psi^{2} \psi^{3}\right\} \\
& G^{1}=2\left\{J^{0} \psi^{1}+\frac{1}{\sqrt{k}}\left(-J^{1} \psi^{0}+J^{2} \psi^{3}-J^{3} \psi^{2}\right)-\frac{2}{\sqrt{k}} \psi^{0} \psi^{2} \psi^{3}\right\}
\end{aligned}
$$

( $J^{0}$ is the current of the Liouville field $\phi$ ) and cyclic permutations for $G^{2}, G^{3}$ we can find [9] the extended $\mathcal{N}=4$ superconformal algebra with $S U(2) \times S U(2) \times U(1)$ symmetry [32]. For $\widetilde{k}=0(k=2)$ the bosonic WZW theory is trivial and we should set $J^{1,2,3}=0$ in (6.7). The effect of the background charge (6.4) can be taken into account by redefining the energy-momentum tensor and supersymmetry charges

$$
\widetilde{T}=T-\frac{1}{\sqrt{k}} \partial J^{0}, \quad \widetilde{G}^{a}=G^{a}-\frac{1}{\sqrt{k}} \partial \psi^{a} .
$$

Now $\widetilde{T}, \widetilde{G}^{a}$ and the level one currents $A_{i}^{-}$satisfy the standard $\mathcal{N}=4$ algebra with $c=6$.

Conformal field theories are characterized not only by their chiral algebra but also by forming a modular invariant using the representations of the chiral algebra. In our case we should find the modular invariants of $\widehat{S U(2)})_{k}$. These are given by an $A-D-E$ classification [26]

$$
\begin{array}{ll}
A_{\widetilde{k}+1} & \widetilde{k} \geq 0 \\
D_{\widetilde{k}} & \widetilde{k} \geq 4, \widetilde{k} \text { even } \\
E_{6} & \widetilde{k}=10 \\
E_{7} & \widetilde{k}=16 \\
E_{8} & \widetilde{k}=28 .
\end{array}
$$

In all cases $\widetilde{k}=k-2=h(G)-2$, where $h(G)$ is the dual Coxeter number of the group $G=A_{\widetilde{k}+1}, D_{\widetilde{\frac{k}{2}+2}}, E_{6,7,8}$. The $A_{\widetilde{k}+1}$ theories are obtained in WZW models on the $S U(2)$ group manifold with level $\widetilde{k}$ and the $D_{\frac{k}{2}+2}$ theories are obtained in WZW theory on the $S O(3)=S U(2) / Z_{2}$ group manifold with level $\widetilde{k}$ [33].

In our case the $U(1)$ gauge theories with $N_{f}$ electrons $\left(A_{N_{f}-1}\right)$ lead to the standard WZW theories on the $S U(2)$ group manifold with level $\widetilde{k}=N_{f}-2$ with its diagonal modular invariant $\left(A_{\widetilde{k}+1}\right.$ in $\left.(6.9)\right)$. Our $S U(2)$ gauge theories with $N_{f}$ quarks $\left(D_{N_{f}}\right)$ lead to a WZW theory on the $S O(3)=S U(2) / Z_{2}$ group manifold with level $\widetilde{k}=2 N_{f}-4$ and hence to the $D_{\frac{k}{2}+2}$ modular invariant in (6.9). Finally, in section 5 we mentioned three 
more theories $E_{6,7,8}$. They are naturally identified with the $E_{6,7,8}$ modular invariants in (6.9).

This provides an explanation of the well known but somewhat mysterious relation between $A-D-E$ groups and modular invariants of $\widehat{S U(2)}$. Our theories with $A-D-E$ global symmetries lead to an $\widehat{S U(2)})_{\bar{k}}$ algebra with $A-D-E$ modular invariants. This is similar to the result of [8,21, where the same theories in three dimensions were studied. There a relation between the $A-D-E$ global symmetries and the singularities in the Coulomb branches was found, thus explaining the relation between the $A-D-E$ groups and $A-D-E$ singularities and discrete subgroups of $S O(3)$ (a closely related relation in the context of string theory had been found in [34]). Finally, if the speculation in the introduction is correct, there can be a direct relation between all these three places where an $A-D-E$ classification exists. The theories with $A-D-E$ global symmetry in two dimensions have a Coulomb branch with an $A-D-E$ modular invariant and a dual description similar to the one in three dimensions with an $A-D-E$ singularity.

We should restate that for $S U(2)$ with $N_{f}=0,1\left(D_{0,1}\right)$ and for $U(1)$ with $N_{f}=1$ $\left(A_{0}\right)$ the description in terms of the tube breaks down. Precisely in these cases there is no Higgs branch, and correspondingly in the string applications there is no enhanced symmetry. It seems that the Coulomb branch for $D_{1}$ is simply $R^{4} / Z_{2}$. It will be interesting to understand the other cases.

\section{Acknowledgments}

We would like to thank P. Aspinwall, T. Banks, M. Douglas, J. Harvey, K. Intriligator, G. Moore, S. Shenker and E. Witten for useful discussions. This work was supported in part by DOE grants \#DE-FG02-90ER40542 and \#DE-FG02-96ER40559. 


\section{References}

[1] O.J. Ganor, D.R. Morrison, N. Seiberg, Nucl.Phys. B487 (1997) 93, hep-th/9610251.

[2] T. Banks, W. Fischler, S. H. Shenker, L. Susskind, Phys.Rev. D55 (1997) 5112, hepth/9610043.

[3] M.J. Duff, R. Minasian, E. Witten, Nucl.Phys. B465 (1996) 413, hep-th/9601036; O.J. Ganor, A. Hanany, Nucl.Phys. B474 (1996) 122, hep-th/9507036; N. Seiberg, E. Witten, Nucl.Phys. B471 (1996) 121, hep-th/9603003.

[4] J. Polchinski, S. Chaudhuri, C.V. Johnson, hep-th/9602052; J. Polchinski, hepth/9611186.

[5] M.R. Douglas, G. Moore, hep-th/9603167; M.R. Douglas, hep-th/9604198.

[6] N. Seiberg, Phys.Lett. B388 (1996) 753, hep-th/9608111.

[7] T. Banks, M.R. Douglas, N. Seiberg, Phys.Lett. B387 (1996) 278, hep-th/9605199.

[8] N. Seiberg, Phys.Lett. B384 (1996) 81, hep-th/9606017.

[9] C.G. Callan, J.A. Harvey, A. Strominger, Nucl.Phys. B359 (1991) 611; C.G. Callan, J.A. Harvey, A. Strominger, hep-th/9112030; S.-J. Rey, In The Proc. of the Tuscaloosa Workshop 1989, 291; Phys. Rev. D43 (1991) 526; S.-J. Rey, In DPF Conf. 1991, 876.

[10] M.R. Douglas, D. Kabat, P. Pouliot, S. Shenker, Nucl.Phys. B485 (1997) 85, hepth/9608024.

[11] M.R. Douglas, hep-th/9512077.

[12] O. Aharony, M. Berkooz, S. Kachru, N. Seiberg, E. Silverstein, hep-th/9707079.

[13] E. Witten, hep-th/9707093.

[14] E. Witten, hep-th/9707121, Strings '95 (World Scientific, 1996).

[15] A. Strominger, Phys.Lett. 383B (1996) 44, hep-th/9512059.

[16] N. Seiberg, hep-th/9705221.

[17] N. Seiberg, Phys. Lett. B318 (1993) 469, hep-ph/9309335.

[18] P. C. Argyres, M. R. Plesser, A. Shapere, Phys. Rev. Lett. 75 (1995) 1699, hepth/9505100.

[19] N. Seiberg and E. Witten, Nucl.Phys. B426 (1994) 19, hep-th/9407087.

[20] N. Seiberg and E. Witten, Nucl.Phys. B431 (1994) 484, hep-th/9408099.

[21] N. Seiberg and E. Witten, In: The Mathematical Beauty of Physics, A Memorial Volume for Claude Itzykson, J.M. Drouffe and J.B. Zuber, Eds., World Scientific, 1997, hep-th/9607163.

[22] A. Sen, Nucl.Phys. B474 (1996) 361, hep-th/9604070; A. Sen, hep-th/9609176; J. Harvey, talk given at the Jerusalem Winter School on Strings and Duality, Jan. 1997.

[23] H. Ooguri, C. Vafa, Nucl.Phys. B463 (1996) 55, hep-th/9511164; D. Kutasov, Phys. Lett B383 (1996) 48, hep-th/9512145; H. Ooguri and C. Vafa, hep-th/9702180.

[24] D. Anselmi, M. Billó, P. Frè, L. Girardello, A. Zaffaroni, Int.J.Mod.Phys. A9 (1994) 3007, hep-th/9304135. 
[25] P. Aspinwall, Phys. Lett. B357 (1995) 329, hep-th/9507012.

[26] A. Cappelli, C. Itzykson, J.B. Zuber, Nucl. Phys. B280 (1987) 445, Comm. Math. Phys. 113 (1987) 1.

[27] S.J. Gates, C.M. Hull, M. Roček, Nucl. Phys. B248 (1984) 157; G.W. Gibbons, G. Papadopoulos, K.S. Stelle, hep-th/9706207.

[28] M.R. Douglas, J. Polchinski and A. Strominger, hep-th/9703031.

[29] M. Roček, K. Schoutens, A.Sevrin, Phys. Lett. B265 (1991) 303; M. Roček, C. Ahn, K. Schoutens, A. Sevrin, hep-th/9110035, Contributed to Workshop on Superstrings and Related Topics, Trieste, Italy, Aug 8-9, 1991, Published in Trieste HEP Cosmol. (1991) 995.

[30] D. Morrison and R. Plesser, hep-th/9508107, Contributed to STRINGS 95: Future Perspectives in String Theory, Los Angeles, CA, 13-18 Mar 1995.

[31] K. Intriligator and N. Seiberg, Phys.Lett. B387 (1996) 513, hep-th/9607207.

[32] A. Sevrin, W. Troost, A. van Proeyen, Phys. Lett. B208 (1988) 601.

[33] D. Gepner and E. Witten, Nucl. Phys. B278 (1986) 493.

[34] E. Witten, Nucl.Phys. B443 (1995) 85, hep-th/9503124. 